\title{
Memoria histórica de la licenciatura en Ciencias de la Educación impartida en la Facultad de Ciencias Humanas de la Universidad Autónoma de Baja California
}

\author{
Historical memory of the Bachelor's degree in Education Sciencias from the \\ Facultad de Ciencias Humanas at Universidad Autónoma de Baja California
}

Juan Carlos Castellanos Ramírez

Shamaly Alhelí Niño Carrasco

\begin{abstract}
RESUMEN
La memoria histórica sobre el desarrollo de las instituciones educativas permite la transferencia cultural y la edificación identitaria de los miembros de la comunidad académica, a la vez es un puente que posibilita la continuidad de un proyecto educativo. En este artículo se presenta un análisis histórico sobre la prospectiva y evolución del programa de Licenciado en Ciencias de la Educación impartido por la Facultad de Ciencias Humanas de la Universidad Autónoma de Baja California, México. Para reconstruir la memoria histórica del programa educativo se llevó a cabo un análisis documental centrado en la revisión de cuatro ejes temáticos: fundamentos epistemológicos de la profesión, prospectiva del campo profesional, institucionalización de la profesión como campo formativo en las universidades y el entorno socio-histórico del programa educativo. Se concluye que el programa educativo analizado tiene sus bases epistemológicas en el pensamiento científicoeducativo promovido en Francia a principios del siglo XX; asimismo, la historia local demuestra la interdisciplinariedad y la flexibilidad curricular como grandes fortalezas del programa educativo.
\end{abstract}

Palabras clave: memoria colectiva, ciencias de la educación, testimonio histórico de las instituciones, análisis prospectivo de programas educativos.

\begin{abstract}
The historical memory on the educational institutions' development allows the cultural transfer and identity building of the members of the academic community, at the same time, it is a bridge that allows the continuity of an educational project. This work presents a descriptive study about the prospective and evolution of the Bachelor's degree in Education Sciences from the Facultad de Ciencias Humanas at Universidad Autónoma de Baja California, Mexico. A documentary analysis was made considering four types of information: epistemological foundations of the profession; prospective of the professional field; institutionalization of the profession as an education field in universities, and the socio-historical context of the educational program. It concludes that the educational program analyzed has epistemological bases on the scientific-educational thought promoted by France at the beginning of the 20th century; likewise, local history of the program points that interdisciplinarity and curricular flexibility are great strengths of the educational program.

Keywords: collective memory, education sciences, historical testimony of the institutions, prospective analysis of educational programs.
\end{abstract}




\section{INTRODUCCIÓN}

Las personas pueden reconstruirse a sí mismas cuando reflexionan sobre sus historias de vida y atribuyen significados a su pasado; Umberto Eco (1999) enfatizó que cuando se pierde la memoria histórica se pierde también la identidad. Bajo esta premisa, la remembranza histórica no solo es importante a nivel individual, sino también en el plano colectivo; de hecho, autores como Herranz y Basabe (1999), Lyons (1996) y Marques, Páez, Valencia y Vincze (2006) han resaltado que la memoria histórica colectiva es vital para asegurar la continuidad de los grupos y para desarrollar el sentimiento de pertenencia a una comunidad. En efecto, el estudio de la memoria histórica puede abordarse desde dos enfoques: el enfoque individual, que remite al hecho de que los recuerdos se encuentran al interior de la mente como parte de un atributo personal, y el enfoque social, en el cual los recuerdos se edifican sobre las experiencias compartidas entre sujetos que transmiten conocimientos y valores para preservar la herencia cultural de una comunidad.

De acuerdo con Mendoza (2005), las instituciones educativas son entidades sociales que se construyen y evolucionan a partir de su historia, por lo tanto, la memoria histórica es uno de los principales mecanismos para la transferencia cultural y la construcción identitaria institucional, ya que, por ejemplo, permite a profesores y estudiantes reconocerse como miembros de una comunidad, ayuda a que las nuevas generaciones se adapten con mayor facilidad y rapidez a la vida institucional o, incluso, favorece la continuidad de un proyecto educativo.

La importancia de dar testimonio escrito sobre los orígenes y el desarrollo de los programas educativos radica, en palabras de Perrupato (2017), en su potencial para "repensarnos a nosotros mismos como agentes institucionales comprendiendo el verdadero alcance de aquello que realizamos" (p. 798). En cambio, cuando la historia de una institución educativa se mantiene en silencio tiende a olvidarse y, a la larga, se corre el riesgo de que pierda su identidad como comunidad (Viñao, 2016).

Juan Carlos Castellanos Ramírez. Profesor-Investigador de Tiempo Completo en la Facultad de Ciencias Humanas de la Universidad Autónoma de Baja California, México. Es doctor en Psicología de la Educación con especialidad en proceso educativos apoyados por TIC, por la Universidad de Barcelona, España. Es miembro del Sistema Nacional de Investigadores (SNI) y colabora con el Cuerpo Académico de Psicología y Educación con clave de registro UABC-CA-155. Forma parte del Comité de Arbitraje de las revistas internacionales Innovación Educativa del Instituto Politécnico Nacional de México y Educare, editada por el Centro de Investigación y Docencia en Educación de la Universidad Nacional de Costa Rica. Correo electrónico: juan. castellanos8@uabc.edu.mx. ID: http://orcid.org/0000-0002-0682-9085.

Shamaly Alhelí Niño Carrasco. Profesora-Investigadora de Tiempo Completo en la Facultad de Ciencias Humanas de la Universidad Autónoma de Baja California, México. Es doctora en Psicología de la Educación, con especialidad en el diseño de herramientas analíticas para el aprendizaje en línea, por la Universidad de Barcelona, España. Es miembro del Sistema Nacional de Investigadores (SNI) y colabora con el Cuerpo Académico de Psicología y Educación con clave de registro UABC-CA-155. Forma parte del Comité de Arbitraje de la revista internacional Información Tecnológica, editada por el Centro de Información Tecnológica de Chile. Correo electrónico: shamaly.nino@uabc.edu.mx. ID: http://orcid.org/000-0002-5623-4418. 
No obstante, el estudio de la historia de las instituciones educativas adquiere características propias, principalmente porque estas son entidades sociales complejas en donde convergen diferentes elementos, por ejemplo, planes y programas de estudio, actores educativos, proyectos, acciones y procesos, entre otros. Como ya se dijo, todos estos aspectos no solo definen la historia institucional, sino que además comprometen a quienes son partícipes de esta historia a proyectar sus significados a través del tiempo.

En este marco, el propósito del presente trabajo consiste en recuperar la memoria histórica del programa educativo de Licenciado en Ciencias de la Educación (LCE) impartido en la Facultad de Ciencias Humanas (FCH) de la Universidad Autónoma de Baja California (UABC). Este programa educativo se ha desarrollado a lo largo de cuatro décadas con el esfuerzo de académicos, administrativos y estudiantes que han luchado por un proyecto académico común.

Hoy en día el programa educativo se enfrenta a un cambio generacional importante, ya que los primeros académicos de dicho programa están cerrando su ciclo como docentes de la institución y entregando la estafeta a una nueva generación de docentes. Bajo este escenario surge el interés de compartir los primeros ciclos de vida del programa educativo, a través de la realización de un estudio histórico-descriptivo orientado por cuatro cuestiones básicas: I) reconocer los fundamentos epistemológicos de las ciencias de la educación, II) identificar la prospectiva de las ciencias de la educación como campo profesional, III) especificar la institucionalización de las ciencias de la educación como campo formativo en las universidades, y IV) describir la evolución del programa educativo de Licenciado de Ciencias de la Educación en la FCH de la UABC.

\section{Metodología}

Se realizó una investigación histórica-descriptiva con el propósito de profundizar en el surgimiento de la profesión en ciencias de la educación y su desarrollo como campo formativo, primero en términos generales y después particularmente dentro de la FCH de la UABC.

Se utilizó la técnica de análisis documental como estrategia de recuperación de la información y reconstrucción de acontecimientos históricos (Dulzaides y Molina, 2004). El análisis documental se desarrolló en cuatro fases que, de acuerdo con Picado y Rico (2011), resultan imprescindibles para la realización de estudios históricos en educación: I) heurística, II) crítica, III) hermenéutica y IV) exposición. En la tabla 1 se muestran las descripciones de cada fase y los criterios específicos adoptados para su concreción en este trabajo. 
Tabla 1. Fases del proceso de análisis documental desarrollado en el estudio.

\begin{tabular}{|c|c|}
\hline Descripción & Criterios adoptados \\
\hline $\begin{array}{l}\text { Heurística: búsqueda, selección y } \\
\text { clasificación de fuentes documentales } \\
\text { que proporcionan testimonio histórico } \\
\text { sobre el tema en cuestión }\end{array}$ & $\begin{array}{l}\text { Para la búsqueda de información se consultaron diversas bases de datos y repositorios } \\
\text { abiertos de literatura académica mediante el cruce y la combinación de diferentes } \\
\text { términos, por ejemplo, "epistemología de las ciencias educativas", "historia de las } \\
\text { ciencias de la educación", "ciencias de la educación en Latinoamérica", "desarrollo de } \\
\text { las ciencias de la educación en México". Como resultado de la búsqueda inicial se } \\
\text { identificaron } 122 \text { artículos }\end{array}$ \\
\hline $\begin{array}{l}\text { Crítica: análisis de los documentos } \\
\text { identificados para asegurar la } \\
\text { autenticidad y utilidad de la información }\end{array}$ & $\begin{array}{l}\text { Para asegurar la autenticidad de la información se consultó el número de referencias } \\
\text { bibliográficas que dan sustento a los documentos y el número de citas asociadas a las } \\
\text { obras. En cambio, la utilidad de la información se determinó a partir de la revisión } \\
\text { de los resúmenes de las obras seleccionadas. Como resultado de este segundo filtro } \\
\text { se eligieron } 26 \text { documentos }\end{array}$ \\
\hline $\begin{array}{l}\text { Hermenéutica: interpretación de } \\
\text { los textos para responder a las } \\
\text { preguntas planteadas en el estudio }\end{array}$ & $\begin{array}{l}\text { De manera individual, tres investigadores revisaron el material seleccionado } \\
\text { centrándose en el análisis de cuatro núcleos de información: I) fundamentos } \\
\text { epistemológicos de las ciencias de la educación; II) prospectiva de las ciencias de la } \\
\text { educación como campo profesional; III) institucionalización de las ciencias de la } \\
\text { educación como campo formativo en las universidades, y IV) el entorno } \\
\text { socio-histórico del programa educativo de Ciencias de la Educación en la } \\
\text { FCH de la UABC y de sus planes de estudio }\end{array}$ \\
\hline $\begin{array}{l}\text { Exposición: selección de los hechos } \\
\text { históricos de mayor relevancia y } \\
\text { organización de los acontecimientos } \\
\text { para su divulgación }\end{array}$ & $\begin{array}{l}\text { Para la elaboración de la memoria histórica se llevaron a cabo cinco sesiones de } \\
\text { trabajo. En la primera sesión se realizó una tabla de referencia cruzada, en la que cada } \\
\text { investigador describió su propia interpretación de los hechos históricos en torno a los } \\
\text { cuatro bloques temáticos definidos en la fase anterior. En las cuatro sesiones restantes } \\
\text { los investigadores establecieron puntos de acuerdo sobre los hechos históricos de } \\
\text { mayor relevancia sobre los que versaría la memoria histórica }\end{array}$ \\
\hline
\end{tabular}

Fuente: Elaboración personal.

\section{Resultados}

El análisis documental ha permitido identificar distintas etapas de consolidación de las ciencias de la educación como profesión y campo formativo. Al respecto, se han organizado los resultados de acuerdo con los cuatro núcleos de información valorados por los investigadores: I) fundamentos epistemológicos de las ciencias de la educación; II) prospectiva de las ciencias de la educación como campo profesional; III) institucionalización de las ciencias de la educación como campo formativo en las universidades, y IV) el entorno socio-histórico del programa educativo de Ciencias de la Educación en la FCH de la UABC. Es preciso señalar que, al tratarse de un estudio descriptivo de corte histórico, los resultados se presentan de manera narrativa; como tal, dentro de la narración se citan las fuentes documentales de referencia. 


\section{Fundamentos epistemológicos de las ciencias de la educación: el debate sobres los estados del conocimiento educativo en las tradiciones alemana, francesa y estadounidense}

La escuela como institución educativa oficial fue uno de los grandes inventos de la comunidad europea a mediados del siglo XVIII (Schriewer, 1991). Los primeros decretos sobre la escuela laica y pública surgieron en Alemania, Francia e Inglaterra (Jover y Thoilliez, 2010), en donde también emergieron distintas posturas epistemológicas sobre los principios, fundamentos y métodos educativos. De hecho, algunos autores (Jover y Thoilliez, 2010; Suasnábar, 2013) señalan que durante los siglos XVIII y XIX el discurso sobre el conocimiento educativo fue objeto de debate desde tres tradiciones distintas: filosófico-educativa, científico-empírica y teórica-práctica.

En relación con la tradición filosófico-educativa (Noguera, 2010), esta se desarrolló con mayor auge dentro de la cultura germánica. Desde esta tradición, la pedagogía se define como una ciencia educativa de carácter teórico y práctico, por lo que su objetivo es reflexionar sobre los fines de la educación y encontrar, dentro de esta reflexión, una racionalidad moral capaz de guiar la práctica de los educadores a través de reglas valorativas y normativas para la práctica pedagógica. La tradición pedagógica alemana tiene sus principios en los postulados filosóficos de Rousseau, Kant, Herbart y Pestalozzi sobre la educación moral y los valores que las personas deben aprender para el bien común, de ahí que surgieran "personajes conceptuales" que representan los principios y creencias pedagógicas del momento como, por ejemplo, el Emilio de Rousseau.

Por su parte, la tradición científico-empírica (Castillejo, 1976; Ríos, 2005; Schriewer, 1991) se desarrolló en Francia entre los años 1960 y 1970 con una gran influencia de la sociología de la educación promovida por Emilio Durkheim. El objetivo fundamental de la tradición francesa fue dotar a la ciencia educativa de procesos metodológicos rigurosos para su racionalidad empírica, a diferencia de la pedagogía, que contaba con una condición teórica y práctica sin ningún sustento de la realidad objetiva. En el fondo, desde la tradición francesa se proponía un abordaje científico de las cuestiones educativas, más allá del sustento filosófico y el carácter práctico que se había promovido desde la pedagogía.

Por lo anterior, en la tradición francesa se utilizó el término "ciencias de la educación" para referirse a las distintas disciplinas que ayudan a explicar los hechos educativos con bases empíricas y, desde entonces, se entienden como un conglomerado pluridisciplinario de todas las ciencias humanas y sociales que centran su objeto de estudio en el ámbito educativo (Castillejo, 1976). Inicialmente, las ciencias de la educación se organizaron en tres bloques de conocimiento: I) disciplinas que investigan los requisitos espacio-temporales de la educación, como demografía pedagógica o economía de la educación, por mencionar algunas; II) disciplinas que 
abordan la relación educativa-fisiología de la educación y su acción, como psicología pedagógica, psicología social y de grupos, entre otras, y III) disciplinas dedicadas a la reflexión teórica y al desarrollo, como planificación del sistema educativo y filosofía de la educación (Jover y Thoilliez, 2010).

En cuanto a la última de las tradiciones, la teórica-práctica (Colom, 1992), esta se desarrolló en el marco de la cultura anglosajona bajo el término "teoría de la educación”. Sus principales exponentes fueron Charles Sanders Peirce, John Dewey y William James, quienes propusieron aplicar las teorías provenientes de distintos campos del conocimiento (ciencias de la educación) para resolver problemas emergentes en el ámbito de la educación formal y no formal. Esta tradición se fue abriendo paso durante los años 70 en las escuelas de pedagogía de Chicago, Nueva York y Columbia, en donde los académicos desarrollaban propuestas de intervención educativa a partir del conocimiento científico obtenido en las ciencias de la educación. En definitiva, la Teoría de la Educación supone una concepción tecnológica de la educación, ya que promueve el desarrollo de conocimiento práctico para resolver problemas emergentes en la sociedad.

Las tres perspectivas epistemológicas antes mencionadas dieron origen a tres campos de formación profesional distintos que, hasta la fecha, siguen vigentes: programas profesionales de pedagogía, ciencias de la educación y educación aplicada.

\section{Prospectiva de las ciencias de la educación como campo profesional en el contexto internacional y nacional}

El principal referente de las ciencias de la educación, como campo del conocimiento, surge en el año de 1967, en Francia, como una crítica a la perspectiva filosófica de la educación que buscaba establecer cómo educar a los ciudadanos para una nación próspera (Avanzini, 2001; Suasnábar, 2013). Hasta finales del siglo XVIII los principios educativos se formulaban bajo la noción pedagógica que, de acuerdo con Durkheim, remiten más a la reflexión en torno a los propósitos de la educación con el fin de generar catálogos prácticos para la enseñanza de los niños (Gargallo, 2009).

No obstante, hacia finales del siglo XIX surgió una creciente desvalorización de la pedagogía a favor de las ciencias de la educación. El principal argumento de las ciencias de la educación fue la necesidad de formular una base científica que ayudara a comprender los procesos educativos para mejorar su funcionamiento y no restringirse solo al tratamiento doctrinal de la enseñanza. La idea de construir una base científica para interpretar los procesos educativos fue apoyada de manera contundente por un grupo de sociólogos y psicólogos reconocidos por su actividad científica en La Sorbona de París (entre ellos Durkehim y Reboul).

Las ciencias de la educación como campo de conocimiento se abrieron al mundo en 1922 a través del libro Sociología de la educación, publicado por Durkheim. En dicha 
obra se sientan las bases epistemológicas de las ciencias de la educación y, a partir de estas, el Estado francés decidió apoyar a las universidades para que ofertaran una carrera profesional coherente con el planteamiento científico de la educación (Forquin, 2003). De esta manera, las ciencias de la educación emergieron en 1967 en el escenario universitario con las primeras facultades e institutos de ciencias de la educación.

A pesar del auge de las ciencias de la educación en Francia, el resto de los países europeos no adoptó dicho planteamiento (por ejemplo Italia, España y Alemania, entre otros) y, en su lugar, la formación pedagógica se elevó a rango universitario. En cambio, la formación de licenciados en ciencias de la educación se adoptó con mayor amplitud en países de América Latina, principalmente en México, Colombia y Argentina (Suasnábar, 2014).

Los campos de formación que inicialmente se establecieron para los programas de licenciatura en ciencias de la educación comprendían el estudio social e histórico de la educación, métodos de investigación aplicados a la educación, filosofía de la educación, demografía pedagógica, economía de la educación, psicología de la educación, sistema educativo nacional y didáctica. A través de los años se han ido precisando los campos disciplinares y también han surgido otras ciencias educativas que responden al contexto actual como lo son, por ejemplo, políticas educativas, economía de la educación, educación intercultural, fisiología de la educación, neuroeducación y tecnología educativa.

\section{Institucionalización de las ciencias de la educación como campo formativo en las universidades}

Las ciencias de la educación como campo formativo y profesional tienen sus orígenes en los primeros cursos técnicos de pedagogía ofrecidos a profesores de secundaria en las universidades francesas de París (1887), Lyon (1884) y Toulose (1887). Sin embargo, fue hasta el año de 1902 cuando Emilio Durkheim dirigió un discurso sobre las bases sociales de la educación (Zambrano, 2004) en la Universidad de París, La Sorbona; en dicho discurso se establecía la necesidad de abordar las cuestiones educativas con una mirada científica, apoyándose para ello de distintas disciplinas humanas y sociales (Jover y Thoilliez, 2010). La postura de Durkheim influyó fuertemente en los cursos de pedagogía ofertados por las universidades, por lo que progresivamente el enfoque filosófico de la educación fue remplazado por la cientificidad aplicada a los procesos educativos (Schriewer, 1991).

En efecto, el 7 de diciembre de 1966, el Ministerio de Educación Nacional de Francia convocó a un grupo de intelectuales -Maurice Debesse, Gaston Mialaret, Paul Fraisse, Jean Chateau y Jean Stoetzel- para crear una licenciatura universitaria en educación. Aunque el Ministerio propuso el título de "Pedagogía" para la impartición del programa, los miembros de la comisión, adeptos a las ideas de Durkheim, 
convencieron a los funcionarios de aprobar la denominación de "Ciencias de la Educación”, bajo el argumento de que la pedagogía se refería solo a cuestiones de formación teórica-práctica, sin ningún enfoque científico que respaldara su estatus universitario (Blanchard-Laville, 2000; Champy-Remoussenard, 2008). Finalmente, el 2 de febrero de 1967, el Ministerio de Educación aprobó los estudios profesionales de Ciencias de la Educación que se impartieron, por primera ocasión, en las universidades de París, Burdeos, Lyon, Caen y Toulouse.

En el contexto mexicano, la primera licenciatura de Ciencias de la Educación de la que se tiene registro se ofertó en 1970 por la Universidad de Monterrey (UDEM), institución privada de inspiración católica. Ocho años después se fundó la Escuela de Ciencias de la Educación en la Universidad Autónoma de Baja California (UABC); años más tarde se convertiría en Facultad de Ciencias Humanas.

El surgimiento de la Escuela de Ciencias de la Educación se enmarca en un contexto de alta afluencia política dentro de la Escuela de Pedagogía de la UABC; en primer lugar, una de las demandas principales por parte de los estudiantes era elevar su propio rango académico, ya que hasta el momento se les subestimaba como "alumnos de una escuela subprofesional, dedicada a preparar maestros para la enseñanza media" (Piñera, 1997, p. 140); en segundo lugar, se vivía un ambiente conflictivo entre el claustro de profesores, quienes se disputaban el liderazgo por la Escuela de Pedagogía.

En este contexto, la idea de plantear un proyecto diferente e innovador no se hizo esperar; fue así como a principios de 1977 se solicitó asesoría al Centro de Didáctica de la Universidad Nacional Autónoma de México (UNAM) para revisar los planes y programas de estudio de la Escuela de Pedagogía. La influencia del pensamiento francés se puso sobre la mesa, ya que una de las primeras consideraciones fue elevar el rango de la profesión, por lo que se requería desarrollar una formación de carácter científico-educativo que permitiera estar al mismo nivel que otras profesiones ofertadas dentro de la universidad. Desde luego, el nuevo proyecto derivó una confrontación entre los académicos adeptos a la pedagogía y los adeptos a las ciencias de la educación. Ante la dificultad de llegar a un acuerdo, se optó por la separación de la Escuela de Pedagogía, de tal manera que en 1978 se fundó la Escuela de Ciencias de la Educación.

\section{El entorno socio-histórico del programa educativo de Ciencias de la Educación en la Facultad de Ciencias Humanas de la UABC}

De acuerdo con la literatura revisada y el testimonio de diversos exalumnos que conforman la primera generación de licenciados en Ciencias de la Educación de la UABC se ha podido recapitular el desarrollo histórico del programa educativo. En concreto, desde la creación del programa hasta la época actual se han identificado cinco etapas 
significativas: I) diseño del primer plan de estudios; II) la reducción de los semestres, los créditos y la organización por áreas formativas en la primera modificación del plan de estudios; III) el establecimiento de la etapa básica y la flexibilidad curricular en la segunda modificación del plan de estudios; IV) cambios en los enfoques curriculares y la implementación de la modalidad semiescolarizada en la tercera modificación del plan de estudios, y V) la implementación de modalidades de aprendizaje no convencionales en la cuarta modificación del plan de estudios.

A continuación se describe cada una de las etapas mencionadas.

\section{Diseño del primer plan de estudios.}

Como se mencionó anteriormente, el primer plan de estudios se desarrolló con el apoyo del Centro de Investigaciones y Servicios Educativos de la Universidad Nacional Autónoma de México (CISE-UNAM). Durante el primer semestre de 1977 la UABC firmó un convenio de colaboración con la UNAM para recibir asesoría del Centro de Didáctica, después Centro de Investigaciones y Servicios Educativos (CISE). El propósito de este proyecto fue revisar los planes y programas de estudio de la Escuela de Pedagogía con el fin de elevar la formación de estudiantes al nivel de licenciatura (Piñera, 1997).

La principal característica del nuevo plan de estudios era la interdisciplinariedad de los distintos campos del conocimiento que se articulaban para explicar los fenómenos educativos. Por lo tanto, la operación del nuevo plan de estudios requería perfiles disciplinares diferentes dentro de la planta de profesores, además de cambios importantes en la organización académica y administrativa existente. La resistencia por aceptar este nuevo planteamiento educativo no se hizo esperar, ya que parte del personal docente argumentaba que esto provocaría el desplazamiento de docentes especializados en pedagogía por profesionales afines a otros campos del conocimiento (por ejemplo sociología, psicología, historia, entre otros).

Por su parte, los profesores que abrazaron la idea del nuevo plan de estudios tomaron la decisión de fundar su propia escuela para operar el programa educativo en Ciencias de la Educación. No obstante, la mayoría del profesorado no había concluido sus estudios de licenciatura, por lo que su formación en las ciencias de la educación era insuficiente para poder desempeñarse con éxito dentro de las aulas (González, 2009).

Para resolver el problema anterior se implementó un programa de nivelación al que asistieron 24 profesores para obtener el título de Licenciado en Ciencias de la Educación. Al concluir con la nivelación de estudios, el 15 de febrero de 1978, las autoridades universitarias tomaron el acuerdo de abrir la nueva Escuela en Ciencias de la Educación (Piñera, 1997). Más tarde, el 23 de febrero de ese mismo año, el rector nombró director provisional de la nueva escuela al licenciado Enrique Pérez 
García, quien estableció los criterios para la conformación de la planta de profesores (Camarena, Heras y Villaseñor, 2017). Con base en dichos criterios, el claustro de profesores se integró con docentes que aprobaron el curso de nivelación y que, por lo tanto, ya contaban con el grado de licenciatura; también se integraron algunos docentes recomendados por el propio Centro de Didáctica de la UNAM.

Finalmente, en marzo de 1978, la creación de la Escuela de Ciencias de la Educación como institución independiente de la Escuela de Pedagogía (Cordero-Arroyo y Salmerón-Castro, 2017) se anunció en los diferentes medios de comunicación del estado de Baja California.

\section{La reducción de los semestres, los créditos y la organización por áreas formativas en la primera modificación del plan de estudios.}

En el marco de las políticas establecidas por el ex-rector de la UABC, Héctor Manuel Gallego García (1983-1987), se modificó el plan de estudios de 1978. Los cambios que se incorporaron se hicieron en tres direcciones (Hirales y Chairez, 2017).

En primer lugar se llevó a cabo la reducción de semestres, de tal manera que la duración del plan de estudios de la licenciatura en Ciencias de la Educación 1986-2 pasó a ser de solo ocho semestres, a diferencia del plan de 1978 que duraba diez semestres. Este nuevo plan contemplaba 46 asignaturas estructuradas en tres áreas académicas: básica, en la que se pretendía dar una visión al estudiante de las disciplinas que se centran en la educación; formativa, que orientaba al estudiante en la adquisición y desarrollo de actitudes, valores, habilidades, conocimientos teóricos, metodológicos e instrumentales correspondientes al área de las ciencias de la educación, y el área aplicativa, que tenía la finalidad de vincular al profesional con la realidad a través de la interpretación y aplicación de lo aprendido.

En segundo lugar, como consecuencia de la depuración de asignaturas dentro del plan de estudios, se efectuó una reducción de créditos dentro de los programas de estudio, por lo que el número total de créditos establecidos para este nuevo plan fue de 389.

En tercer lugar se realizó una nueva organización curricular, de tal manera que las materias se articularon por áreas formativas. Otro cambio importante remite a la organización del mapa curricular por campos disciplinares como: administración y planeación, investigación, psicología educativa, sociología educativa, tecnología educativa, administración instrumental y ciencias sociales.

Finalmente, el registro del nuevo plan de estudios se llevó a cabo en mayo de 1986 con los tres cambios sustanciales que ya se han comentado, mientras la licenciada Clara Elena Gallegos Salas fungía como directora de la Escuela de Ciencias de la Educación. 


\section{El establecimiento de la etapa básica y la flexibilidad curricular en la segunda modificación del plan de estudios.}

La segunda modificación del plan de estudios se llevó a cabo en 1993, durante el periodo rectoral del doctor Luis Llorens Báez (1991-1994). Las características principales de este plan remiten, por un lado, a la interdisciplinariedad de los distintos campos del conocimiento pertenecientes a las ciencias humanas y, por otro lado, a la flexibilidad curricular (Gallegos y Gallegos, 2017).

Sobre la primera característica, en este plan se optó por establecer una etapa básica que abarcaba dos semestres. Durante este periodo los estudiantes de las licenciaturas de Psicología, Comunicación, Sociología y Ciencias de la Educación compartían asignaturas introductorias al estudio de las ciencias humanas. Con ello se pretendía que los estudiantes establecieran un primer contacto con todas las opciones profesionales, para que en el momento de iniciar el área disciplinaria lo hicieran con un sustento vocacional.

Por su parte, durante la etapa disciplinaria y terminal, los estudiantes tenían la posibilidad de cursar materias obligatorias y optativas, de tal manera que adquirieran el conocimiento básico e imprescindible de las ciencias de la educación y que, además, tuvieran la posibilidad de desarrollar perfiles profesionales de acuerdo con sus propios intereses.

Sobre la segunda característica, la flexibilidad curricular, este plan le daba al estudiante la posibilidad de seleccionar su carga académica en función de sus necesidades y de avanzar, con ello, en la obtención de los créditos; así, por ejemplo, el estudiante podía aumentar o disminuir su carga académica en determinado semestre ante posibles problemas o circunstancias adversas durante su formación.

Debido a la propia naturaleza del plan se crearon instancias académicas para poder operarlo, como el Consejo Académico por área, Centro de Apoyo Docente, Sistema de Consejería Académica, Comisión de ingreso, permanencia y promoción del alumnado, Comisión de prácticas profesionales y de servicio social, entre otros. De la misma manera aparecieron otras modalidades de aprendizaje, como son la cátedra colegiada, tutorías para estudios independientes, ejercicio investigativo y/o práctica académica, así como cursos expositivos. Cabe destacar que, a razón de estos cambios, en 1996 la Escuela de Ciencias de la Educación pasó a ser la Facultad de Ciencias Humanas.

\section{Cambios en los enfoques curriculares y la implementación de la modalidad semiescolarizada en la tercera modificación del plan de estudios.}

Durante la administración rectoral del doctor Alejandro Mungaray Lagarda (20022006) se realizaron cambios importantes en los enfoques curriculares de los planes 
y programas de estudio ofertados por la UABC. El propósito fundamental consistió en realizar un cambio de paradigma, por lo que se transitó de un enfoque curricular basado en objetivos a un enfoque basado en competencias (López y Reyes, 2017).

En este contexto, la Facultad de Ciencias Humanas abanderó dicho proyecto a través del programa de licenciatura en Ciencias de la Educación 2003 (Rodríguez, Reyes y Lizárraga, 2013). El tránsito entre paradigmas no fue tarea sencilla, ya que implicaba transformar tanto las prácticas de enseñanza tradicional basadas en la cátedra lineal como las actividades de aprendizaje de los estudiantes basadas en la memorización.

Gracias al compromiso de la planta de profesores y su propia inquietud intelectual para llevar a la práctica los nuevos enfoques curriculares, dentro del plan de estudios se definió, por primera vez, un perfil de egreso en el que se declararon ocho competencias básicas que recogían la intención de formar profesionistas con posibilidades de intervenir en espacios formales y no formales, para apoyar procesos de enseñanza y de aprendizaje presenciales y no presenciales.

Asimismo, el proyecto del plan de estudios del 2003 continuó apoyando la flexibilidad del programa educativo y, además, se incorporó una modalidad semiescolarizada dentro de la oferta educativa. Originalmente dicha modalidad se pensó para el público adulto que, por distintas circunstancias, no disponía del tiempo suficiente para asistir a las aulas de manera regular entre semana. A diferencia del programa escolarizado, la modalidad semiescolarizada propuso un menor número de horas de aprendizaje en el aula y, en cambio, se otorgó mayor peso a las horas de autoaprendizaje y aprendizaje guiado mediante asesorías académicas puntuales.

\section{Implementación de modalidades de aprendizaje no convencionales en la cuarta modificación del plan de estudios.}

En el año 2012 se llevó a cabo la última modificación del plan de estudios de Ciencias de la Educación, y a la fecha este plan continúa ofertándose. El nuevo proyecto se fundó en el marco de las teorías educativas emergentes centradas en la formación de estudiantes mediante la práctica guiada; desde esta perspectiva se buscó ampliar las experiencias de aprendizaje más allá de las actividades en el aula. En concreto, en el nuevo plan de estudios se incluyeron créditos optativos bajo el nombre de modalidades de aprendizaje no convencionales (UABC, 2012).

La primera de estas modalidades es el Ejercicio investigativo y requiere el seguimiento por parte de un profesor investigador, pues se busca que los estudiantes desarrollen habilidades para la investigación a través de la realización de un estudio empírico sobre problemas educativos actuales. Esta opción formativa es elegible a partir del sexto semestre, dado que está orientada, fundamentalmente, para que los estudiantes desarrollen la tesis de grado. 
Otra modalidad de aprendizaje no convencional es la Ayudantía docente; su finalidad es que los estudiantes consoliden sus habilidades para la enseñanza, mediante el apoyo a los profesores del propio programa educativo. En efecto, bajo la supervisión sistematizada de un profesor, los estudiantes colaboran en la elaboración de los planes de clase, material didáctico y pruebas para la evaluación de los aprendizajes, mismas que son puestas en práctica frente a grupo.

Una tercera modalidad es la Ayudantía de investigación; con esta, los estudiantes se incorporan en alguno de los proyectos de investigación registrados en la FCH. Todos los estudiantes que se encuentren inscritos pueden elegir esta opción formativa, independientemente del semestre que estén cursando.

Finalmente, como cuarta modalidad de aprendizaje no convencional, el Proyecto de vinculación permite que los estudiantes se relacionen directamente con empresas, organizaciones o dependencias gubernamentales afines al perfil profesional de la carrera. El propósito es que el estudiante lleve a la práctica los conocimientos adquiridos en el aula y desarrolle nuevas habilidades sobre la profesión, con la ayuda de profesores activos. De esta manera se intenta que el estudiante enriquezca su formación con la propia experiencia y pericia de los miembros de la comunidad profesional en la que se inserta.

Además de lo anterior, en este plan de estudios también se da seguimiento a la flexibilidad curricular a través de la oferta de materias en línea, así como la interdisciplinariedad entre distintos campos profesionales, en donde los estudiantes tienen la oportunidad de cursar materias optativas ofertadas en otros programas educativos de la misma institución, siempre y cuando la naturaleza de las materias aporte contenidos básicos para la profundización del conocimiento educativo, la enseñanza y el aprendizaje.

\section{Conclusiones}

Como se ha constatado en este trabajo, la educación escolar ha sido históricamente un tema de reflexión desde diferentes enfoques y tradiciones epistemológicas del conocimiento educativo (filosófico-educativo, científico-educativo, entre otras). En lo que corresponde a las ciencias de la educación, estas se centran en abordar científicamente los problemas educativos desde diferentes perspectivas metodológicas provenientes de las ciencias humanas. Entre todos los países que han sido precursores de las ciencias de la educación, Francia es el país que se distingue por los grandes aportes realizados tanto a nivel epistemológico como a nivel de campo formativo.

Sobre este último nivel, las ciencias de la educación se han adoptado positivamente dentro de las universidades latinoamericanas como campo de formación, mientras que en Europa siguen predominando las carreras de Pedagogía y Educación para maestros. 
A partir de 1967, cuando en Francia se crearon las primeras licenciaturas en ciencias de la educación, la implementación en México se hizo en un periodo relativamente corto. En el caso de la UABC, el programa educativo de Licenciado en Ciencias de la Educación comenzó a operar en 1978, es decir, once años después de su creación en Francia. Este programa se ha sustentado en cinco planes de estudio distintos que han respondido a las necesidades regionales y nacionales del momento. Además, los modelos curriculares también se han ido modificando de acuerdo con los paradigmas educativos y políticas nacionales vigentes, como lo fueron el plan de estudios por etapas disciplinares basado en objetivos, el plan de estudios por áreas de formación, el plan de estudios flexible, el plan de estudios por competencias y, finalmente, el plan de estudios de orientación práctica.

Actualmente, las principales fortalezas de la licenciatura en Ciencias de la Educación de la UABC se remiten a dos aspectos. Por una parte, la naturaleza interdisciplinaria de las ciencias de la educación ha permitido la vinculación con otros campos formativos ofrecidos dentro de la misma universidad, en detalle, hay una amplia gama de asignaturas optativas que se ofrecen a los estudiantes de educación desde otras licenciaturas, ampliando el conocimiento educativo desde múltiples enfoques, y además les permite compartir experiencias de aprendizajes con estudiantes de otros campos profesionales.

Por otra parte, la flexibilidad curricular ofrece distintos espacios de aprendizaje no convencionales para que los estudiantes aprendan directamente a través de la práctica guiada en contextos educativos reales (ejercicios investigativos, ayudantías docentes, ayudantías de investigación, proyectos de vinculación y prácticas profesionales). Asimismo, el programa educativo actual sobresale por la flexibilidad que ofrece en cuanto a las modalidades educativas, en donde los estudiantes pueden elegir diversas materias optativas impartidas de manera presencial, híbrida y/o en línea.

Para concluir este trabajo es importante subrayar tres consideraciones con respecto a las implicaciones del estudio. En primer lugar, los resultados han permitido reconocer los fundamentos epistemológicos de las ciencias de la educación, aportando con ello información relevante en torno al debate sobre los estados del conocimiento educativo, particularmente en las tradiciones alemana, francesa y estadounidense.

En segundo lugar, si bien las ciencias de la educación han sido objeto de análisis profundos desde el punto de vista del ejercicio profesional, todavía existe poca literatura sobre su desarrollo histórico como campo formativo en las universidades; en este sentido, el recorrido histórico realizado sobre las ciencias de la educación como programa formativo es un precedente importante para reconstruir la historia de las ciencias de la educación en México y es, al mismo tiempo, un llamado para que otras universidades en las que se oferta dicho programa educativo resguarden su historia institucional para las futuras generaciones de estudiantes y profesores. 
En tercer lugar, el recorrido histórico que se ha realizado en torno a los planes de estudios con los cuales ha operado el programa educativo de Licenciado en Ciencias de la Educación, ofertado por la Facultad de Ciencias Humanas en la UABC, recupera momentos trascendentales en la vida académica de la institución educativa que, ante el inminente cambio generacional de profesores, permite dar continuidad a un proyecto educativo con más de cuatro décadas de operación.

Tomar en cuenta las tres consideraciones anteriores y sus implicaciones epistemológicas e identitarias para las futuras modificaciones del programa educativo de Licenciado en Ciencias de la Educación sin duda refuerza el interés histórico y metodológico de este trabajo; por una parte, la recuperación de la memoria histórica colectiva (Herranz y Basabe, 1999; Lyons, 1996; Marques et al., 2006) del programa permitirá que esta se transfiera a las nuevas generaciones, promoviendo la construcción identitaria institucional a la que se refiere Mendoza (2005); por otra parte, el análisis documental realizado por fases (Dulzaides y Molina, 2004; Picado y Rico, 2011) ha permitido reconstruir los acontecimientos históricos de la institución educativa y del propio programa, pero se reconoce, como parte de las limitaciones del trabajo, la necesidad de aplicar otras técnicas e instrumentos de recuperación de los datos, con el fin de profundizar en la relación de esa memoria histórica colectiva con el sentimiento de pertenencia institucional.

\section{REFERENCIAS}

Avanzini, G. (2001). El desarrollo de las "ciencias de la educación" y los fundamentos del renacimiento de la reflexión filosófica en el campo de la educación en Francia. Pensamiento Educativo. Revista de Investigación Educacional Latinoamericana, 28(1), 51-66. Recuperado de: http://pensamientoeducativo.uc.cl/index.php/ $\mathrm{pel} /$ article/view/187/398.

Blanchard-Laville, C. (2000). De la co-disciplinarité en sciences de l'Education. Revue Française de Pédagogie, 132, 55-66. Recuperado de: https:/ /www.jstor.org/ stable/41201594.

Camarena Fuentes, A., Heras Modad, R. y Villaseñor Amézquita, G. (2017). Plan de estudios de la Licenciatura en Ciencias de la Educación formulado en 1978 (manuscrito inédito, Facultad de Ciencias Humanas, Universidad Autónoma de Baja California, México).

Castillejo Brull, J. L. (1976). Nuevas perspectivas en las ciencias de la educación: Pedagogía general 1. Madrid, España: Anaya.
Champy-Remoussenard, P. (2008). Regards croisés depuis et sur les Sciences de l'Education. Recherches \& éducations, 1, 9-26. Recuperado de: https://journals. openedition.org/rechercheseducations/432.

Colom Cañellas, A. J. (1992). El saber de la teoría de la educación. Su ubicación conceptual. Teoría de la Educación. Revista Interuniversitaria, 4, 11-19. http:// doi.org/10.14201/teri.2940.

Cordero-Arroyo, G. y Salmerón-Castro, A. M. (2017). El servicio profesional docente y las licenciaturas en Ciencias de la Educación. Revista Iberoamericana de Educación Superior, 8(23), 3-24. http://doi.org/10.22201/ iisue.20072872e.2017.23.243.

Dulzaides Iglesias, M. y Molina Gómez, A. (2004). Análisis documental y de información: dos componentes de un mismo proceso. Revista Cubana de Información en Ciencias de la Salud, 12(2), 1. Recuperado de http:// scielo.sld.cu/scielo.php?script=sci_arttext\&pid =S1024-94352004000200011. 
Eco, U. (1999). Preámbulo. ¿Sólo puede construirse el futuro sobre la memoria del pasado? En F. BarretDucrocq (ed.), ¿Por qué recordar? (pp. 183-186). Barcelona: Granica.

Forquin, J. C. (2003). Une discipline pour la République: La Science de l'éducation en France 1882-1914. Éducation et sociétés, 12(2), http:/ / doi.org/163-168.10.3917/ es.012.0163.

Gallegos Santiago, M. y Gallegos Santiago, E. (2017). Plan de estudios flexible de la Licenciatura en Ciencias de la Educación, 1993 (manuscrito inédito, Facultad de Ciencias Humanas, Universidad Autónoma de Baja California, México).

Gargallo López, B. (2009). La teoría de la educación. Objeto, enfoques y contenidos. Teoría de la Educación. Revista Interuniversitaria, 14, 19-46. http://doi. org/10.14201/ted.2967.

González, M. (2009). Universidad Autónoma de Baja California: memoriay testimonio de universitarios 1957-1997. Mexicali, B. C.: Universidad Autónoma de Baja California.

Herranz, K. y Basabe, N. (1999). Identidad nacional, ideología política y memoria colectiva. Psicología Politica, 18, 31-47. Recuperado de: https://www.uv.es/ garzon/psicologia\%20politica/N18-3.pdf.

Hirales Pacheco, M. y Chairez Jiménez, M. (2017). Reestructuración del Plan de estudios de la Licenciatura en Ciencias de la Educación, 1986 (manuscrito inédito, Facultad de Ciencias Humanas, Universidad Autónoma de Baja California, México).

Jover, G. y Thoilliez, B. (2010). Cuatro décadas de teoría de la educación: ¿una ecuación imposible? Teoría de la Educación. Revista Interuniversitaria, 22(1), 43-64. http:/ / doi.org/10.14201/ted.7131.

López, E. y Reyes Estrada, J. (2017). Plan de estudiospor competencia de la Licenciatura en Ciencias de la Educación, 2003 (manuscrito inédito, Facultad de Ciencias Humanas, Universidad Autónoma de Baja California, México).

Lyons, E. (1996). Coping with social change: Processes of social memory in the reconstruction of identities. En G. Breakwell y E. Lyons (eds.), Changing European identities. Social psychological analyses of social change (pp. 31-39). Oxford: Elsevier.

Marques, J., Páez, D., Valencia, J. y Vincze, O. (2006). Effects of group membership on the transmission of negative historical events. Psicología Política, 32,
79-105. Recuperado de: https://www.uv.es/garzon/ psicologia\%20politica/N32-5.pdf.

Mendoza García, J. (2005). Exordio a la memoria colectiva y el olvido social. Athenea digital: Revista de Pensamiento e Investigación Social, 8, 1-26. http://doi. org/10.5565/rev/athenead/v1n8.217.

Noguera Ramírez, C. E. (2010). La constitución de las culturas pedagógicas modernas: una aproximación conceptual. Pedagogía y Saberes, 33, 9-25. https://doi. org/10.17227/01212494.33pys9.25.

Perrupato, S. (2017). Pensar la historia de las instituciones educativas en la Argentina: aportes para el debate. Revista Latinoamericana de Ciencias Sociales, Niñez, y Juventud, 15(2), 797-806. http://doi.org/10.11600/1 692715x.1520102082016.

Picado, M. y Rico, L. (2011). La selección de libros de texto en una investigación histórica en educación matemática. Epsilon, 28(1), 99-112. Recuperado de https://thales.cica.es/epsilon/sites/thales.cica. es.epsilon/files/\%5Bfield_volumen-formatted $\% 5 \mathrm{D} /$ revista77_7.pdf.

Piñera, D. (1997). Historia de la Universidad Autónoma de Baja California, 1957-1997. Mexicali, BC: Universidad Autónoma de Baja California.

Pont, E. (1983). El pedagogo y las ciencias de la educación en Francia: conversación con el profesor Gastón Mialaret. Educar, 3, 183-185. Recuperado de: https:// ddd.uab.cat/pub/educar/0211819Xn3/0211819Xn 3p183.pdf.

Ríos Beltrán, R. (2005). Las ciencias de la educación: entre universalismo y particularismo cultural. Revista Iberoamericana de Educación, 36(4), 1-15. https://doi. org/10.35362/rie3642806.

Rodríguez Díaz, P., Reyes Sánchez, O. y Lizárraga Aguilar, A. (2013). El desempeño profesional del licenciado en Ciencias de la Educación de la Facultad de Ciencias Humanas de la UABC. Un estudio de caso. Revista Iberoamericana para la Investigación y el Desarrollo Educativo, 10. Recuperado de: http://ride.org.mx/1-11/index. $\mathrm{php/RIDESECUNDARIO/article/view/243/238.}$

Suasnábar, C. (2013). La institucionalización de la educación como campo disciplinar: un análisis desde la perspectiva de la historia social de las ciencias sociales. Revista Mexicana de Investigación Educativa, 18(59), 1281-1304. Recuperado de: https://www.comie.org. 
$\mathrm{mx} /$ revista/v2018/rmie/index.php/nrmie/article/ view/289/289.

Suasnábar, C. (2014). Tradición e internacionalidad: Archivos de Ciencias de la Educación (3ra. época) y el pensamiento educacional en los años sesenta. Archivos de Ciencias de la Educación, 8(8), 1-23. Recuperado de: https://www.archivosdeciencias.fahce.unlp.edu.ar/ article/view/Archivos08a05/pdf_109.

Schriewer, J. (1991). La construcción de la pedagogía científica. Diferenciación institucional y disciplinar. Funciones formativas y praxis educativa en la ciencia universitaria de la educación en Alemania y Francia. Revista de Educación, 296, 137-174. Recuperado de: https:// sede.educacion.gob.es/publiventa/d/482/19/0.
Universidad Autónoma de Baja California (UABC) (2012). Proyecto de modificación del programa de Licenciatura en Ciencias de la Educación. Recuperado de http:// fch.mxl.uabc.mx/wp-content/uploads/2015/04/ proyecto-modificacion-educacion.pdf.

Viñao Frago, A. (2016). La historia de la educación como disciplina y campo de investigación: viejas y nuevas cuestiones. Espacio, Tiempo y Educación, 3(1), 21-42. http://doi.org/10.14516/ete.2016.003.001.3.

Zambrano Leal, A. (2004). El lugar de la pedagogía en las ciencias de la educación en Francia. Revista Colombiana de Educación, 47. https://doi. org/10.17227/01203916.5516.

Cómo citar este artículo:

Castellanos Ramírez, J. C., y Niño Carrasco, S. A. (2020). Memoria histórica de la licenciatura en Ciencias de la Educación impartida en la Facultad de Ciencias Humanas de la Universidad Autónoma de Baja California. IE Revista de Investigación Educativa de la REDIECH, 11, e732. doi: https://doi.org/10.33010/ie_rie_rediech.v11i0.732. 